\title{
Designing a Decision Support System for Production Scheduling Task in Complex and Uncertain Manufacturing Environments
}

\author{
Emrah Arica ${ }^{1}$, Jan Ola Strandhagen ${ }^{1}$, and Hans-Henrik Hvolby ${ }^{2}$ \\ ${ }^{1}$ Department of Production and Quality Engineering, NTNU, Norway \\ ${ }^{2}$ Centre for Logistics, Aalborg University, Denmark \\ emrah.arica@ntnu.no
}

\begin{abstract}
The production planning and control process is performed within complex and dynamic organizations made up of equipment, people, information, IT systems, and influenced by a multitude of external factors. How to effectively schedule in uncertain and complex manufacturing environments, still remains a central question to academics and practitioners. In this paper, we propose a framework that can be utilized to design/enhance decision support systems for scheduling activities in complex and uncertain manufacturing environments. The framework is based on the analysis of the relevant literature that addressed human, organizational, and technological aspects of the production planning and scheduling.
\end{abstract}

Keywords: production scheduling and control, complex and uncertain manufacturing environments, decision support system.

\section{Introduction}

Today, a rich amount of scientific literature exists, addressing the planning and scheduling task in uncertain manufacturing environments by proposing advanced methods, techniques, and algorithms that can predict and/or react on unscheduled events [see for example the recent literature reviews provided by 1, 2-5]. Majority of the theoretical studies view this task from a technical perspective with well-defined, simplified and objective-driven analytical problems [6, 7], incorporating a limited number of events and their estimated or identified time impact on single schedules $[1,2,5]$.

In stable and reliable environments, these analytical tools and computerized systems can yield expected performance results, especially for the high level material and capacity planning decisions [3]. However, shop types with high uncertainty and complexity where many factors affect the decision making process can be substantially challenging [8-12]. As such, very few of the advanced models and systems that address dynamic scheduling/ re-scheduling decisions have influenced and provided guidelines in the industrial practice $[2,13,14]$. This results in substantial efforts for customizing the scheduling systems to the actual needs of the production environment and decision makers [15].

B. Grabot et al. (Eds.): APMS 2014, Part I, IFIP AICT 438, pp. 589-596, 2014.

(C) IFIP International Federation for Information Processing 2014 
Thus, the basic reason behind this theory and practice gap can be attributed to the inconsistency between the theoretical definition of the dynamic scheduling process and the complexity of this process in practice. Several field-based studies investigated and captured the important characteristics of the scheduling and control practice in order to reduce this gap and improve the applicability and implementation of the decision support models and tools [e.g. 9, 16-22]. These practice-based studies showed that the scheduling activity is driven by unscheduled events in practice; involving a multitude of aspects (e.g. human, organizational, and technological - HTO) in the decision making process to identify and solve the problems effectively, besides the technical aspect. These upcoming insights might flesh out a holistic view on important requirements of designing decision support systems for effective scheduling/re-scheduling decisions. This view is not clear as these field-based reports and contributions addressed different elements of this process in isolation, based on different objectives, methodologies and methods (e.g. hierarchical task analysis, cognitive task analysis). Depending on the objective and the chosen methodology, they pointed out various characteristics and requirements of the decision making process.

The overall aim of this paper is to develop a framework for guiding the design/enhancement of a decision support system for dynamic scheduling decisions, utilizing the contributions and linkages in related literature. While the design of current scheduling systems are mainly focusing on generating the initial schedules with a top-down approach, this framework implies that the design/enhancement of decision support system should start with a bottom-up approach. More specifically, we focus on the dynamics of the event handling and rescheduling process and emerging HTO aspects around the information needs to make effective informed decisions. The impact of the characteristics of this process on the design of a decision support system is examined and reflected. As such, the applicability and practicality of the system can be enhanced, addressing the needs of the production control situation and decision makers.

\section{Dealing with Unscheduled Events in a Typical IT Configuration}

The majority of the modern manufacturing firms use Enterprise Resource Planning (ERP) systems for production planning and control activities [21]. The fundamental benefits of the ERP systems come from their abilities to process and record transactions efficiently, rather than their inherent planning and control capabilities [23]. When used for production planning and control, ERP systems deploy the Material Requirements Planning (MRP) logic [24]. The MRP translates the MPS into timephased net material requirements and calculates the Planned Order Release (POR) for the detail scheduling and execution use on the manufacturing shop floor. At the shopfloor, the generated POR schedule is exposed to uncertainties that the system is not accountable for. This creates many problems for the production execution later on the shop-floor, such as varying workloads and changing bottlenecks [25].

Over the past two decades, APS systems have emerged to supplement the ERP systems and eliminate some of the major MRP assumptions [26]. APS has by far outperformed the planning and scheduling functionality of the ERP system by for example 
considering the limited resource capacities simultaneously with the material planning function. A strong feature in APS is the ability to simulate different planning scenarios before plan release [27], which leads to meeting the customer order deadlines with most efficient capacity planning solutions. By doing that, they prevent some of the shop floor uncertainties that ERP systems are bound to encounter on the shop floor. APS is recently standardized and presented as an advanced scheduling technology and how this technology can handle uncertainties is still an open research topic [12].

Manufacturing Execution Systems (MES) have been evolved to aid production execution, monitoring, and control activities on the shop floor, closing the drawbacks of the ERP systems in real-time detailed information exploitation from the shop floor. MES can be described as shop floor information and communication systems that provide feedback on a real-time basis [28]. The functionalities of MES cover the manufacturing execution and control tasks in an enterprise, making the shop floor data available and measuring the real time performance indicators (e.g. equipment utilization, inventory availability, quality status). This makes it a powerful tool to detect and identify the disturbances. However, MES especially lacks decision making capabilities, more specifically in evaluation and diagnosis of what re-planning and rescheduling actions should be taken [29].

\section{The Decision Making Process and Associated Systems}

The results of number of field-based reports indicate that taking event-driven rescheduling decisions is an iterative problem identification and solution process, performed in a continuum of activities with information gathering and interactions between different parties in the organization [e.g. 7, 16, 17, 21]. The scope and dynamics (e.g. how to identify and solve the problem, what information is needed, who and what systems should be involved, what actions can be taken) of this decision making process depends on the situational factors (e.g. a machine breakdown happened on a bottleneck machine).

As such, the basic decision making process to handle unscheduled events is to detect, identify, evaluate, and response (see figure 1). Once the cause of the event is detected via automated systems or humans [30], the decision maker(s) should identify the significance of the problem(s) within given situation in order to classify what needs an immediate attention and who should be involved [9]. At the next stage, the alternative re-scheduling solutions are evaluated in order to find an appropriate one in the given situation and constraints. This evaluation stage also involves communication and information sharing efforts across the organization (e.g. operators, schedulers, planners, and even the sales department in some cases) in order to see what constraints can be relaxed, such as what jobs can be delayed [21]. Once the appropriate solution is chosen and actions are taken, schedulers then deal with the consequences. This post-analysis stage may also result in further planning adjustments (e.g. changes in executional priorities of jobs).

Hence, the decision making process involves multiple functional roles (e.g. PPC, maintenance, quality, sales) and IT systems in the problem identification and solving process. There can be many different configurations, applications, and functionality of ERP, APS, and MES systems, supporting different functional processes and roles in 
an organization in varying extents and contents. But, in an abstract level, possible support of these systems in the event handling and rescheduling process can be given based on the previous section where we discussed a typical setting. However, the realization of these benefits still suffers from the complexity of the re-scheduling process in practice for different reasons, in terms of an effective decision making process. Many of these challenges can be gathered around the paradigm of information needs which may encompass several issues explained in the following framework. The framework also indicates the HTO aspects involved to acquire the required information in the decision making process.

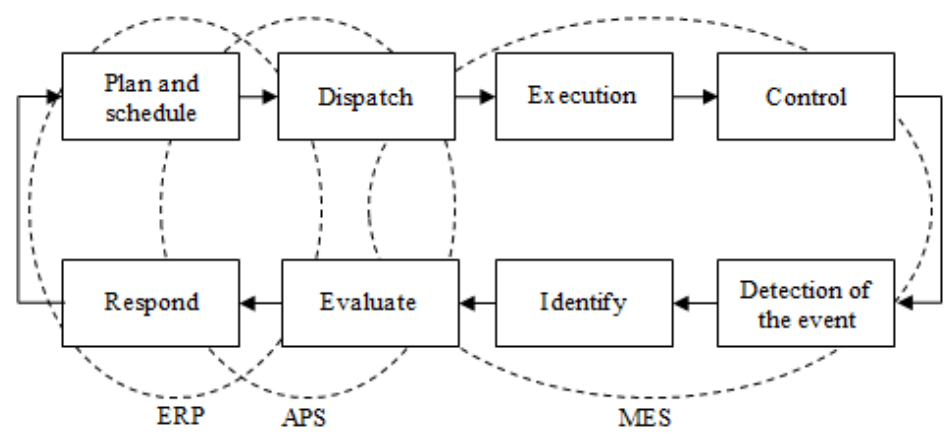

Fig. 1. Decision-making loop for responding unscheduled events and major support areas of the systems

\section{$4 \quad$ Framework}

As mentioned above, we have formulated the requirements of the decision support systems around the information needs that emerge when unscheduled events occur. Many of the HTO aspects are involved in the problem identification and solution process because of the efforts to incorporate the right information into the decisions.

\subsection{Information Representation/Modelling}

This issue relates to the information introduced and represented in the modelled version of scheduling/re-scheduling task in the APS system. With varying degrees, mathematical models make assumptions and define the problem space with lesser complexity than actually it is [10]. This results in part of the internal or external constraint information to be left out of the model, which leads to a perceived problem space. When changes are detected, the adjustments are still done in the perceived problem space which leads to a new perceived problem. However, depending on the type of event and the situation, these changes causes some of the "un-represented" constraints (i.e. internal or external) to be considered in the decision making process. As such, new information is needed to identify the problem and solve in given situation. Let's consider a simple fictional example of machine breakdown that disturbs a local schedule. To take a re-allocation action, the decision maker need to get 
additional information about the situation such as status of the alternative machines that can run the disturbed jobs, status of the required additional resources to run the new operation (e.g. operator, tools, other consumables), internal and external status of other jobs sharing the alternative machine (e.g. their schedule, inventory levels, and priority status). This example can be widened with much more situational information to identify the problem (constraints) and find an appropriate solution.

This issue brings up different interrelated HTO aspects in the problem identification and solution process. The human schedulers deal with this ill-defined situation by experience, knowledge, and communication and feedback efforts to reach at informed decisions [19, 21, 31]. Furthermore, during this communication and feedback process, they acquire part of the required information from the existing IT systems [20]. A related aspect is the division of the decision making autonomy. This aspect can be considered in two dimensions; (i) division of autonomy between shop floor personnel and schedulers [11] (ii) division of autonomy between humans and tools [32]. It is very difficult to implement the advanced scheduling rules and systems, if there is no agreement on the division of autonomy, namely who should decide what [11]. The multiple case study of Ivert [12] also confirms the importance of this issue for successful implementation of the APS in the type of shop floors where uncertainty is high and many options exist to deal with them.

\subsection{Information Availability}

This issue relates to the availability of the required information in the IT systems to identify and solve the problem. As the occurrence of the event entails a need for additional information to solve the episode in a given context effectively, the availability of the information becomes a salient characteristic of this process [33]. Most of the information might be available in the systems, however, in this respect, the use of IT systems may be challenged with the availability of the information in an updated form. In a periodic control decision, this prerequisite wouldn't be a problem to put in place as the aggregated information (e.g. inventory levels at the end of the week) is captured and used. However, the event-driven decision making is a demanding process in terms of the need for detailed updated information about both the primary (e.g. the current inventory levels of the disturbed jobs with the machine breakdown) as well as the secondary constraints (e.g. the current inventory levels of the other jobs sharing the alternative machine) of the problem. Jonsson and Mattson [34] argue that accurate lead times and safety stocks are two of the most critical parameters for achieving high planning and control performance. Furthermore, poor data quality is one of the main causes of the use of specific tools and spreadsheets to complement the scheduling tools [33].

\subsection{Information Accessibility}

This issue relates to the integration of the other systems with the scheduling/rescheduling system in order to retrieve and exploit the required information in the decision making process. A key factor to implement such an event-driven control mechanism is providing the information at the time of perceived information need 
[35]. In many cases the information may be available in different systems and formats, which makes it difficult to access it when needed [36]. In this respect, some of the authors proposed automated solutions that utilizes the real time information from other systems and technologies [e.g. 14]. Authors that follow the practice-based research stream mainly favored the human-computer interactive solutions [e.g. 32]. Whether it will be processed by the algorithm or visualized as a supportive function, the integration of the information sources to the scheduling system becomes a relevant issue. In this context, Framinan and Ruiz [33] emphasize that integration of systems may become too complex and impossible and suggests simple and modular approaches such as using data exchange protocols for information exchange.

\subsection{Information Structuring and Visualization}

This issue relates to how information is presented to the decision maker. The studies show that most of the IT systems have drawbacks to take into consideration of the cognitive skills of the people. As this is an information-driven process, the effectiveness of the decisions also relies on how quick the decision maker can make the sense of the situation from the available information. Today a great deal of real time information is available with recent technological advancements [30]. However, besides the advantage of improved possibility for decision making with more available and accurate information, a challenge of dealing with the increased data in a practical way arises. Hence, visualization also plays an important role in the successful implementation of systems. The use of planning support computer programs is device dependent with psychological relevance but yet event independent [37]. In general, the person who needs to interact with the program and the real time information are disregarded. Having a better perception of the planning system for the scheduler needs a better availability and visibility of the required information, based on the cognitive analysis of the human scheduler [38]. The limits of human decision making skills should be well understood when designing the display content.

\section{Conclusions}

This study provided indications to the design of the decision support systems for production scheduling task in complex and uncertain manufacturing environments. The motivation behind this study was to build a framework that can facilitate operationalization of the important HTO aspects derived from field-based studies addressing the gap between scheduling theory and practice. There is need for a further study to explicitly reflect the outlined requirements on the design and architecture of a scheduling system and test it in a real life case study for a validity check.

Acknowledgements. This study was financed by the research center called The Norwegian Manufacturing Future (SFI NORMAN). 


\section{References}

1. Vieira, G.E., Herrmann, J.W., Lin, E.: Rescheduling manufacturing systems: A framework of strategies, policies, and methods. Journal of Scheduling 6(1), 39-62 (2003)

2. Aytug, H., et al.: Executing production schedules in the face of uncertainties: A review and some future directions. European Journal of Operational Research 161(1), 86-110 (2005)

3. Mula, J., et al.: Models for production planning under uncertainty: A review. International Journal of Production Economics 103, 271-285 (2006)

4. Ouelhadj, D., Petrovic, S.: A survey of dynamic scheduling in manufacturing systems. Journal of Scheduling 12(4), 417-431 (2009)

5. Hozak, K., Hill, J.A.: Issues and opportunities regarding replanning and rescheduling frequencies. International Journal of Production Research 47(18), 4955-4970 (2009)

6. Higgins, P.G.: Interaction in hybrid intelligent scheduling. International Journal of Human Factors in Manufacturing 6(3), 185-203 (1996)

7. Crawford, S., et al.: Investigating the work of industrial schedulers through field study. Cognition, Technology \& Work 1(2), 63-77 (1999)

8. Wiers, V.C.S., Van Der Schaaf, T.W.: A framework for decision support in production scheduling tasks. Production Planning \& Control 8(6), 533-544 (1997)

9. McKay, K.N., Buzacott, J.A.: The application of computerized production control systems in job shop environments. Computers in Industry 42(2), 79-97 (2000)

10. McKay, K.N., Wiers, V.C.S.: Integrated decision support for planning, scheduling, and dispatching tasks in a focused factory. Computers in Industry 50(1), 5-14 (2003)

11. Wiers, V.C.: The relationship between shop floor autonomy and APS implementation success: evidence from two cases. Production Planning and Control 20(7), 576-585 (2009)

12. Ivert, L.K.: Shop floor characteristics influencing the use of advanced planning and scheduling systems. Production Planning \& Control 23(6), 452-467 (2012)

13. Kreipl, S., Pinedo, M.: Planning and scheduling in supply chains: An overview of issues in practice. Production and Operations Management 13(1), 77-92 (2004)

14. Georgiadis, P., Michaloudis, C.: Real-time production planning and control system for jobshop manufacturing: A system dynamics analysis. European Journal of Operational Research 216(1), 94-104 (2012)

15. Pinedo, M.L.: Planning and Scheduling in Manufacturing and Services, 3rd edn. Springer, Berlin (2007)

16. Jackson, S., Wilson, J.R., MacCarthy, B.L.: A new model of scheduling in manufacturing: Tasks, roles, and monitoring. Human Factors: The Journal of the Human Factors and Ergonomics Society 46(3), 533-550 (2004)

17. McKay, K.N., Wiers, V.C.S.: Planning, scheduling and dispatching tasks in production control. Cognition, Technology \& Work 5(2), 82-93 (2003)

18. Berglund, M., Karltun, J.: Human, technological and organizational aspects influencing the production scheduling process. International Journal of Production Economics 110(1), 160-174 (2007)

19. Cegarra, J.: A cognitive typology of scheduling situations: A contribution to laboratory and field studies. Theoretical Issues in Ergonomics Science 9(3), 201-222 (2008)

20. Karltun, J., Berglund, M.: Contextual conditions influencing the scheduler's work at a sawmill. Production Planning and Control 21(4), 359-374 (2010)

21. de Snoo, C., et al.: Coordination activities of human planners during rescheduling: Case analysis and event handling procedure. International Journal of Production Research 49(7), 2101-2122 (2011) 
22. Webster, S.: A case study of scheduling practice at a machine tool manufacturer. In: MacCarthy, B.L., Wilson, J.R. (eds.) Human Performance in Planning and Scheduling, pp. 6781. Taylor \& Francis, London (2001)

23. Powell, D., Riezebos, J., Strandhagen, J.O.: Lean production and ERP systems in smalland medium-sized enterprises: ERP support for pull production. International Journal of Production Research 51(2), 395-409 (2013)

24. Koh, S., Saad, S.: A holistic approach to diagnose uncertainty in ERP-controlled manufacturing shop floor. Production Planning \& Control 14(3), 273-289 (2003)

25. Chen, K., Ji, P.: A mixed integer programming model for advanced planning and scheduling (APS). European Journal of Operational Research 181(1), 515-522 (2007)

26. Steger-Jensen, K., et al.: Advanced planning and scheduling technology. Production Planning \& Control 22(8), 800-808 (2011)

27. Hvolby, H.-H., Steger-Jensen, K.: Technical and industrial issues of Advanced Planning and Scheduling (APS) systems. Computers in Industry 61(9), 845-851 (2010)

28. Manetti, J.: How technology is transforming manufacturing. Production and Inventory Management Journal 42(1), 54-64 (2001)

29. De Ugarte, B.S., Artiba, A., Pellerin, R.: Manufacturing execution system-a literature review. Production Planning and Control 20(6), 525-539 (2009)

30. Cowling, P., Johansson, M.: Using real time information for effective dynamic scheduling. European Journal of Operational Research 139(2), 230-244 (2002)

31. McKay, K.N., Wiers, V.C.S.: The human factor in planning and scheduling. In: Herrmann, J.W. (ed.) Handbook of Production Scheduling, pp. 23-57. Springer Science+Business Media, Inc., New York (2006)

32. Van Wezel, W., Hoc, J.M., Cegarra, J.: Allocating Functions to Human and Algorithm in Scheduling. In: Fransoo, J.C., Waefler, T., Wilson, J.R. (eds.) Behavioral Operations in Planning and Scheduling, pp. 339-370. Springer, Berlin (2011)

33. Framinan, J.M., Ruiz, R.: Guidelines for the deployment and implementation of manufacturing scheduling systems. International Journal of Production Research 50(7), 1799-1812 (2012)

34. Jonsson, P., Mattsson, S.-A.: Inventory management practices and their implications on perceived planning performance. International Journal of Production Research 46(7), 1787-1812 (2008)

35. Tang, O., Naim, M.: The impact of information transparency on the dynamic behaviour of a hybrid manufacturing/remanufacturing system. International Journal of Production Research 42(19), 4135-4152 (2004)

36. Leung, Y., Choy, K., Kwong, C.: A real-time hybrid information-sharing and decision support system for the mould industry. The Journal of High Technology Management Research 21(1), 64-77 (2010)

37. Wezel, W., Cegarra, J., Hoc, J.M.: Allocating Functions to Human and Algorithm in Scheduling. In: Behavioral Operations in Planning and Scheduling, pp. 339-370 (2011)

38. Riezebos, J., van Wezel, W.: Planner-oriented design of algorithms for train shunting scheduling. Planning in Intelligent Systems: Aspects, Motivations, and Method, 477-496 (2006) 